\title{
The learning curve associated with imageless navigation in total knee arthroplasty
}

\author{
Michelle A. Zabat ${ }^{1}$ (D) $\cdot$ Christian T. Oakley ${ }^{1} \cdot$ Scott E. Marwin ${ }^{1} \cdot$ Morteza Meftah $^{1} \cdot$ Ran Schwarzkopf $^{1}$
}

Received: 11 September 2021 / Accepted: 25 January 2022 / Published online: 28 February 2022

(c) The Author(s), under exclusive licence to Springer-Verlag GmbH Germany, part of Springer Nature 2022

\begin{abstract}
Introduction Computer-assisted navigation systems (CAS) are increasingly being integrated into total knee arthroplasty (TKA) procedures, but perceptions of associated learning curve and increased operative time continue to curtail uptake. Newer-generation navigational systems aim to streamline integration into surgical workflow to mitigate increases in operative time. Here, we assess the impact of a novel imageless CAS on operative time for TKA.

Methods A retrospective analysis of prospectively collected data of a cohort of patients undergoing primary unilateral TKA with one of three surgeons between October 2019 and March 2020 was conducted. Consecutive cases using a novel imageless CAS were included in analysis. For each surgeon, average operative time was recorded and compared in sequential five-case cohorts to average operative time for the same procedure performed conventionally using a two-tailed $t$ test.

Results Average conventional operative times were 95.9 $\pm 15.0,86.6 \pm 13.7$, and $116.9 \pm 25.1 \mathrm{~min}$ for the three surgeons. Initial CAS-assisted operative times increased to 107.0 $\pm 9.8(p=0.07)$ and 102.4 $\pm 13.2(p=0.06)$ min for Surgeons 1 and 2 and decreased to $113.2 \pm 9.8 \mathrm{~min}(p=0.52)$ for Surgeon 3. Most recent CAS-assisted operative times were 94.8 \pm 13.9 $(p=0.88), 88.7 \pm 15.3(p=0.84)$, and 104.8 $\pm 13.2(p=0.12) \mathrm{min}$ as compared to pre-CAS. Absolute differences for the most recent navigated procedures ranged from $12.1 \mathrm{~min}$ faster to $2.0 \mathrm{~min}$ slower.

Conclusion The learning curve for TKA navigation may be as few as 10 cases, and any associated increases in operative time may be transient and non-significant. Moreover, navigation may ultimately speed operative time, perhaps as the result of enhanced intraoperative assessment of alignment.
\end{abstract}

Keywords Computer-assisted navigation $\cdot$ Patient reported outcomes $\cdot$ Navigated TKA

\section{Introduction}

Total knee arthroplasty (TKA) is an effective surgical intervention for end stage knee joint osteoarthritis, as well as one of the most common surgical procedures performed in the United States [1-3]. This number is expected to increase exponentially in the coming decades, with demand projected to rise by $673 \%$ to 3.48 million procedures per year in 2030 [4]. Since the first use of computer-assisted navigation systems (CAS) in TKA in 1997, utilization of computer navigation has slowly gained traction, with $7.0 \%$ of U.S. TKA procedures utilizing computer navigation in 2014 [5, 6]. The

Ran Schwarzkopf

Ran.Schwarzkopf@nyulangone.org

1 Department of Orthopaedic Surgery, NYU Langone Orthopedic Hospital, NYU Langone Health, 301 East 17th Street, New York, NY 10003, USA original aim of CAS was to improve component alignment during TKA, as proper alignment has been demonstrated to correlate with improved stability and clinical scores as well as lower rates of loosening [7, 8]. Conversely, malalignment has been associated with increased post-operative pain and risk of implant wear and loosening, contributing to risk of revision surgery and patient dissatisfaction [9-12]. Prior studies have demonstrated the ability of CAS to achieve operative alignment goals more consistently than conventional TKA [6, 13-16].

Despite the advantages of CAS on implant alignment in TKA, uptake remains curtailed by a handful of perceived concerns. One is the potential for increased operative time, which has been detailed in prior studies [16-20]. Increased operative time may result in increased operating costs, especially when taken alongside installation, training, and maintenance costs associated with surgical technology. In addition, it may put patients at risk for higher rates of 
complications [21]. However, it is also well-established that new surgical techniques or technology are broadly associated with a learning curve which may manifest as increased operative time [22-26]. More research is needed on whether any operative time increases are inherent to a particular surgical technology or if they are transient increases associated with an expected learning curve, as well as whether any persistent operative time increases are of clinical significance.

Though prior studies have examined the learning curve associated with robotic technology for total knee and hip arthroplasty, to our knowledge no recent study has been performed regarding the learning curve for CAS-assisted TKA using longitudinal, surgeon-specific, prospectively collected cohort data. In this study, we aim to assess the learning curve and impact on operative time associated by contrasting operative times of three joint reconstructive surgeons for non-navigated vs. navigated TKA with a novel imageless CAS.

\section{Methods}

\section{Data collection}

This was a retrospective study of prospectively collected data including patients 18 years and older undergoing TKA between October 2019 and March 2020 at a single urban orthopedic specialty hospital by one of three senior fellowship-trained and board-certified surgeons with more than 10 years of experience. The imageless navigation system used in this study (Intellijoint KNEE ${ }^{\mathrm{TM}}$; Intellijoint Surgical Inc., ON, Canada) was introduced at the study center in October 2019; as such, the included cases were the first CAS-assisted procedures performed by the three surgeons. The study period was ended in March 2020 to avoid confounding of primary outcomes by COVID-19 protocols. Patients who underwent bilateral TKA, revision TKA, or non-elective TKA for trauma, tumor, or other reasons were excluded. All patients went through a mandatory preadmission clearance, underwent a TKA education program, and were stratified to appropriate discharge disposition. All patients included in this study participated in our institutional comprehensive total joint pathway program, which encompasses uniform standardized protocols for all aspects of perioperative care. A standard institutional postoperative rehabilitation protocol is followed for all patients at our institution. All data were extracted from our institution's electronic data warehouse (Epic Caboodle, Version 15, Verona, WI) using Microsoft SQL Server Management Studio 2017 (Redmond, WA).

All procedures were performed via the medial parapatellar approach and used the same imageless CAS. Given this navigation device accommodates either a femur- or tibia-first workflow, the TKA procedures included in this study represent both workflows. The device is composed of a camera, optical trackers, and a computer workstation that sits outside of the sterile field and is controlled by the surgeon via buttons on the camera. The workflow for tibial cuts is as follows: after the primary incision and exposure of the femur and tibia, a bone screw is drilled into the tibia and the optical bone tracker is attached. The bone screw may be inserted either on the articular or extra-articular surface of the proximal tibia; however, for cut-verification, extra-articular placement of the screw is required. The tibia is registered using the optical probe tracker by defining the medial and lateral malleoli, the tibial centre, the anteroposterior (AP) axis, and the medial and lateral tibial plateaus. The optical probe tracker is then attached to the cutting guide, providing real-time feedback of the prospective cut measurements. Cutting guide position can be adjusted to the desired orientation and then secured in place. Post-resection, the tracker is placed on the cut surface and the bone cut is measured by the navigation device (Fig. 1).

The workflow for femoral cuts is similar to that for tibial cuts: a bone screw is drilled into the femur and the optical bone tracker is attached. Again, while the bone screw can be inserted either on the articular or extra-articular surfaces of the distal femur, cut verification requires extra-articular positioning of the screw. The femur is registered by rotating the hip joint through a range of motion to capture the hip centre of rotation. The optical probe tracker is used to define the femur centre, Anterior-posterior femoral line, and the lateral and medial femoral condyles. As with the tibia, the optical probe tracker is attached to the cutting guide, providing realtime feedback to help position the cutting guide, which is secured with pins once the desired orientation is achieved. Post-resection, the tracker is placed on the cut surface and the bone cut is measured by the navigation device (Fig. 1).

During the period from introduction of the CAS to March 2020, 75 total and 64 navigated cases were performed by surgeon 1, 36 total and 33 navigated cases were performed by surgeon 2 , and 52 total and 30 navigated cases were performed by surgeon 3 . There were no selection criteria for use of navigation by the surgeons only the limited access to the technology in the early period of its use. For each of the surgeons, each navigated case was chronologically grouped into cohorts of 5 cases per cohort. This yielded 13 cohorts for surgeon 1, 7 cohorts for surgeon 2, and 6 cohorts for surgeon 3 . These cohorts were created to serve as multiple time intervals at which patients could be compared [26]. Consecutive non-navigated cases performed by each surgeon immediately prior to the introduction of CAS served as a control group of size equal to the number of cases included 
A.
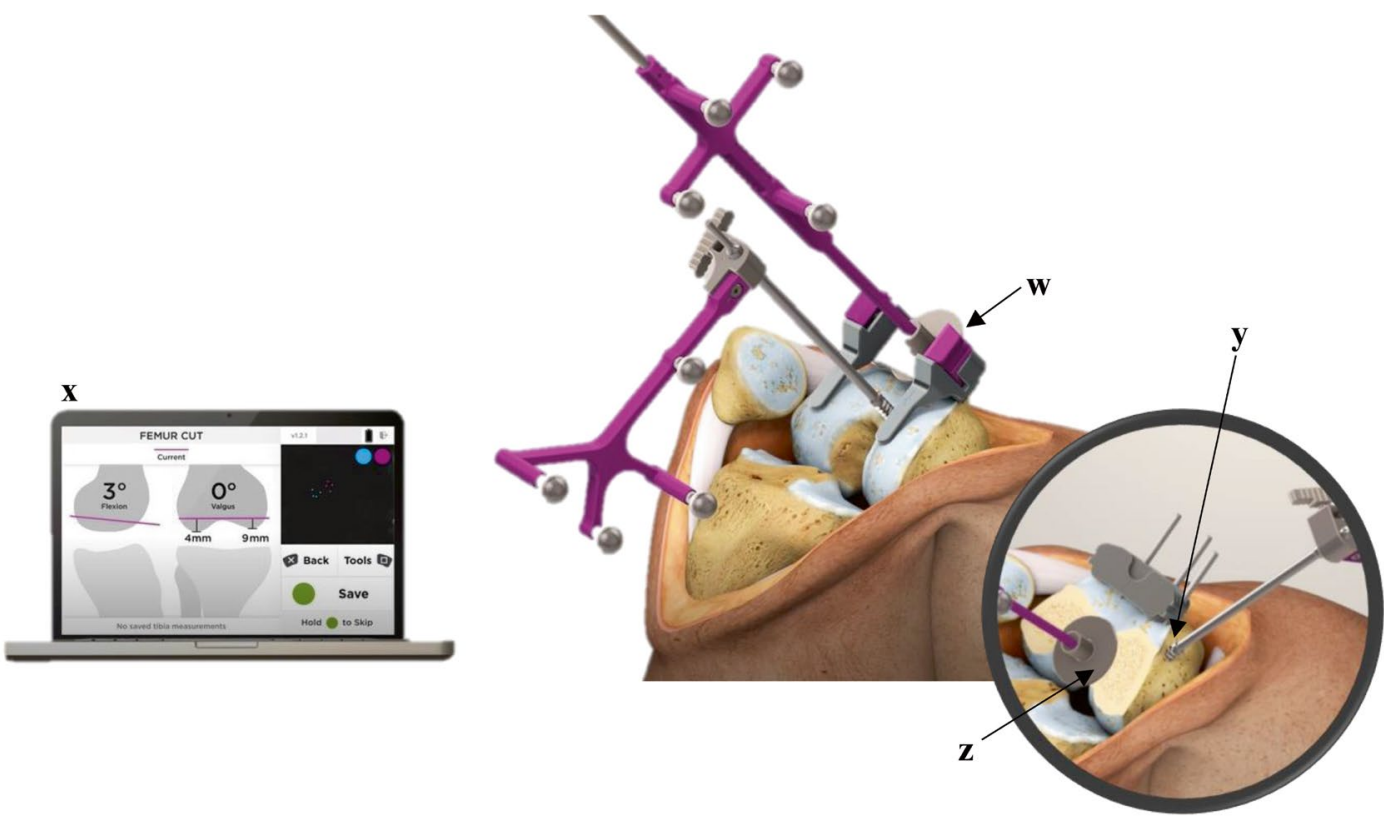

B.

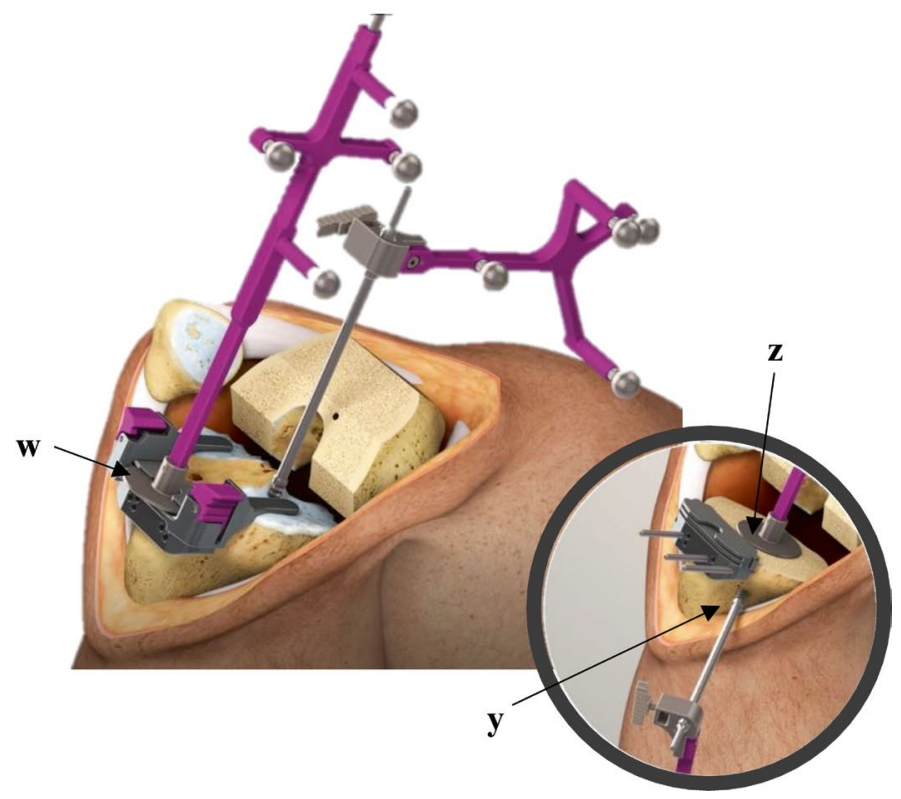

Fig. 1 Imageless computer navigation device. A With optical probe tracker slotted into the femoral cutting guide (w) changes in guide position are detected by the camera (not pictured) and the impact on planned cut parameters are displayed on the workstation $(\mathrm{x})$, in real time. Insert shows that with extra-articular installation of the bone screw (y) the probe tracker can be placed on femur post-resection (z) and bone cut parameters are displayed on the workstation, in

in the study group for each surgeon. For both the study and the control group, operative time was calculated as the time the incision was made to the time the incision was closed, reported in minutes. real time. B With optical probe tracker slotted into the tibial cutting guide $(w)$ changes in guide position are detected by the camera (not pictured) and the impact on planned cut parameters are displayed on the workstation $(\mathrm{x})$, in real time. Insert shows that with extra-articular installation of the bone screw (y) the probe tracker can be placed on tibia post-resection ( $\mathrm{z}$ ) and bone cut parameters are displayed on the workstation, in real time

\section{Statistical analysis}

For each surgeon, the mean operative time for each cohort was calculated and charted temporally. The first cohort and last cohort of navigated cases were compared with each other and with the control group of non-navigated procedures 
using two-sided $t$ tests. All descriptive data are represented as means \pm standard deviation. All statistical analyses were performed using Microsoft Excel for Mac (Version 16.50, Microsoft, Redmond, WA). A $p$ value of less than 0.05 was considered to be statistically significant.

\section{Results}

Within the time period of interest, there were 64,33 , and 30 cases meeting inclusion criteria performed by each of the three surgeons included in the study. Average operative time for non-navigated primary unilateral TKA was $95.9 \pm 15.0 \mathrm{~min}, 86.6 \pm 13.7 \mathrm{~min}$, and $116.9 \pm 25.1 \mathrm{~min}$.

\section{Surgeon 1}

Results from surgeon 1 yielded a mean operative time of $100.1 \pm 11.0$ (range 95.6-104.7) minutes across all of the thirteen sequential navigated cohorts. For the first cohort of procedures using the novel imageless CAS, average operative times were $107.0 \pm 9.8 \mathrm{~min}$. $(p=0.07)$ as compared to $95.9 \pm 15.0 \mathrm{~min}$. for non-navigated (Table 1). Average operative time for the most recent cohort of procedures performed with the CAS was $94.8 \pm 13.9 \mathrm{~min}$. $(p=0.88)$ as compared to $95.9 \pm 15.0 \mathrm{~min}$. for non-navigated (Table 2). Between the first and the most recent CAS-assisted cases, average operative time was not significantly different $(p=0.20)$ (Table 3 ). Absolute average difference in operative time from the nonnavigated control procedures to the most recent procedure cohort was $1.2 \mathrm{~min}$ faster with navigation. Operative times over the course of the study period are represented in Fig. 2.

\section{Surgeon 2}

Results from surgeon 2 yielded a mean operative time of $97.8 \pm 16.7$ (range 85.2-111.0) minutes across all of the seven sequential navigated cohorts. For the first cohort of procedures using the novel imageless CAS, average operative times were $102.4 \pm 13.2 \mathrm{~min}$. $(p=0.06)$ as compared to $86.6 \pm 13.7 \mathrm{~min}$. for non-navigated (Table 1). Average operative time for the most recent cohort of procedures performed with the CAS was $88.7 \pm 15.3 \mathrm{~min} .(p=0.84)$ as compared to $86.6 \pm 13.7 \mathrm{~min}$. for non-navigated (Table 2). Between the first and the most recent CAS-assisted cases, average operative time was not

Table 1 First CAS-assisted TKA vs. non-navigated TKA

\begin{tabular}{lcll}
\hline & Control & First cohort & $p$ value \\
\hline Surgeon 1 & $95.9 \pm 15.0 \mathrm{~min}$ & $107.0 \pm 9.8 \mathrm{~min}$ & 0.07 \\
Surgeon 2 & $86.6 \pm 13.7 \mathrm{~min}$ & $102.4 \pm 13.2 \mathrm{~min}$ & 0.06 \\
Surgeon 3 & $116.9 \pm 25.1 \mathrm{~min}$ & $113.2 \pm 9.8 \mathrm{~min}$ & 0.52 \\
\hline
\end{tabular}

Table 2 Most recent CAS-assisted TKA vs. non-navigated TKA

\begin{tabular}{lrrr}
\hline & Control & Most recent & $p$ value \\
\hline Surgeon 1 & $95.9 \pm 15.0 \mathrm{~min}$ & $94.8 \pm 13.9 \mathrm{~min}$ & 0.88 \\
Surgeon 2 & $86.6 \pm 13.7 \mathrm{~min}$ & $88.7 \pm 15.3 \mathrm{~min}$ & 0.84 \\
Surgeon 3 & $116.9 \pm 25.1 \mathrm{~min}$ & $104.8 \pm 13.2 \mathrm{~min}$ & 0.12 \\
\hline
\end{tabular}

significantly different $(p=0.27)$ (Table 3$)$. Absolute average difference in operative time from the non-navigated control procedures to the most recent procedure cohort was $2.0 \mathrm{~min}$ slower with navigation. Operative times over the course of the study period are represented in Fig. 2.

\section{Surgeon 3}

Results from surgeon 3 yielded a mean operative time of $111.0 \pm 10.8$ (range 104.8-13.2) minutes across all of the six sequential navigated cohorts. For the first cohort of procedures using the novel imageless CAS, average operative times were $113.2 \pm 9.8 \mathrm{~min}$. $(p=0.52)$ as compared to $116.9 \pm 25.1 \mathrm{~min}$. for non-navigated (Table 1). Average operative time for the most recent cohort of procedures performed with the CAS was $104.8 \pm 13.2 \mathrm{~min}$. $(p=0.12)$ as compared to $116.9 \pm 25.1 \mathrm{~min}$ for non-navigated (Table 2). Between the first and the most recent CAS-assisted cases, average operative time was not significantly different $(p=0.29)$ (Table 3$)$. Absolute average difference in operative time from the non-navigated control procedures to the most recent procedure cohort was $12.1 \mathrm{~min}$ faster with navigation. Operative times over the course of the study period are represented in Fig. 2.

\section{Combined cases}

When the first two navigated cohorts, the last two navigated cohorts, and the non-navigated control cohorts from surgeons 1,2, and 3 were combined to create three cohorts, a trend similar to those of the single-surgeon analyses was observed. The mean operative time for the earliest navigated cases was $104.1 \pm 10.4 \mathrm{~min}$., while the mean operative time for the most recent navigated cases was $102.0 \pm 14.7 \mathrm{~min}$., showing a decrease in operative time that did not reach the level of significance $(p=0.53)$ (Table 4$)$. The mean operative time for the control cohort was $99.8 \pm 17.9$ min., which

Table 3 First CAS-assisted TKA vs. most recent CAS-assisted TKA

\begin{tabular}{llcl}
\hline & Cases 1-5 & Most recent & $p$ value \\
\hline Surgeon 1 & $107.0 \pm 9.8 \mathrm{~min}$ & $94.8 \pm 13.9 \mathrm{~min}$ & 0.20 \\
Surgeon 2 & $102.4 \pm 13.2 \mathrm{~min}$ & $88.7 \pm 15.3 \mathrm{~min}$ & 0.27 \\
Surgeon 3 & $113.2 \pm 9.8 \mathrm{~min}$ & $104.8 \pm 13.2 \mathrm{~min}$ & 0.29 \\
\hline
\end{tabular}




\section{Operative Time for Navigated TKA}

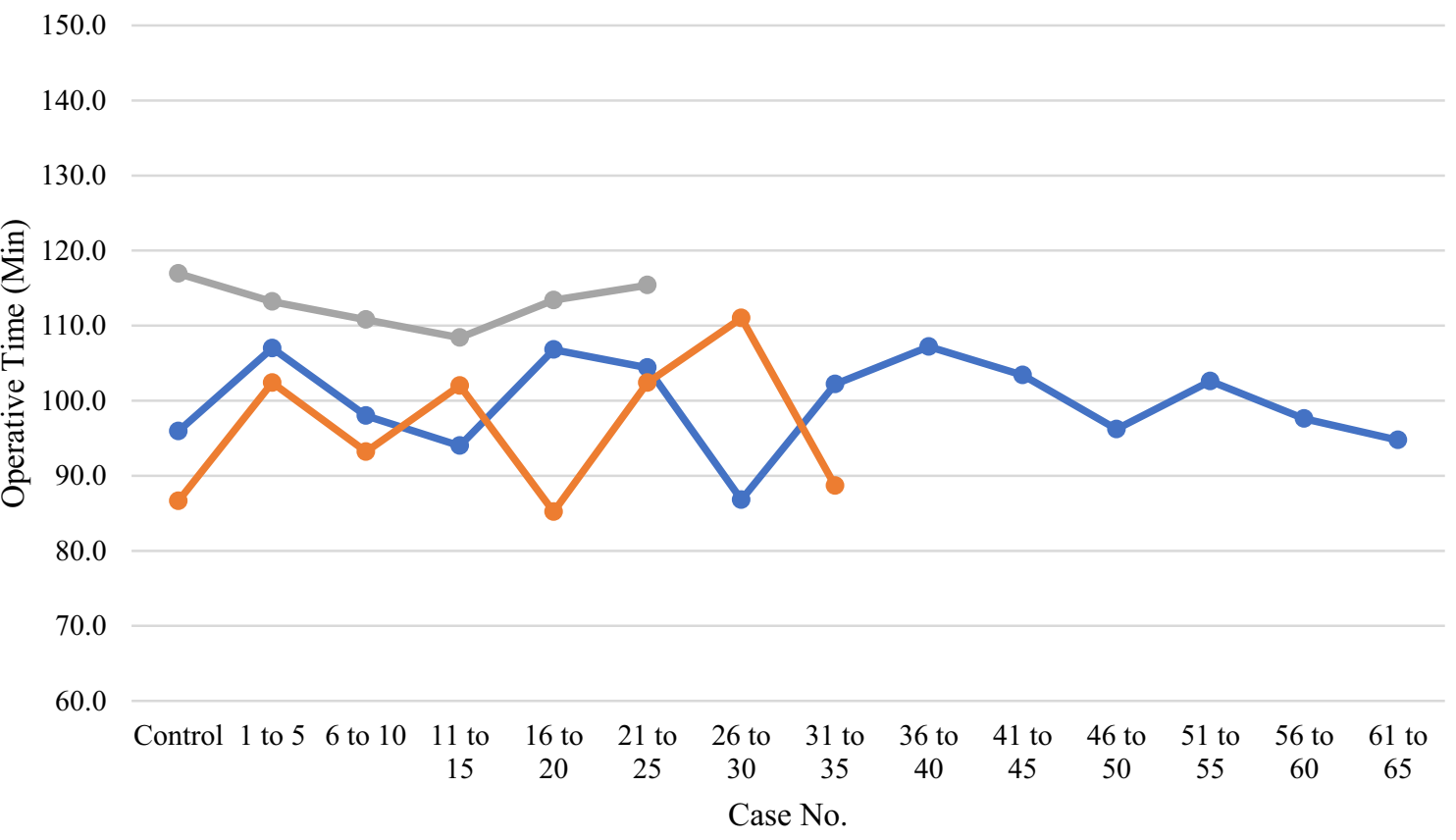

Fig. 2 Sequential Operative Times for TKA Procedures Using a Novel Imageless CAS

Table 4 Initial CAS-assisted TKA vs. most recent CAS-assisted TKA for combined cohorts

\begin{tabular}{llc}
\hline $\begin{array}{l}\text { Initial navigated combined } \\
\text { cohort }\end{array}$ & $\begin{array}{l}\text { Most recent navigated com- } \\
\text { bined cohort }\end{array}$ & $p$ value \\
\hline $104.1 \pm 10.4 \mathrm{~min}$ & $102.0 \pm 14.7 \mathrm{~min}$ & 0.53 \\
\hline
\end{tabular}

was slightly faster than the initial navigated cohort $(p=0.20)$ and the most recent navigated cohort $(p=0.55)$ (Table 5).

\section{Discussion}

Computer navigation has proven to improve implant alignment and component positioning when used as part of TKA. However, greater understanding of the impact on operative time and associated learning curve of CAS is needed, especially regarding novel systems which have not previously been assessed. The aim of the present study was to analyze trends in operative time for TKA performed with a novel imageless CAS as compared to non-navigated TKA on a surgeon-specific basis. Our findings highlight the experiences of three surgeons with the introduction of imageless CAS into the surgical workflow and indicate that the associated learning curve may not be as pronounced as has been previously hypothesized.

Previous studies assessing operative time of navigated vs. conventional TKA have produced mixed results, with some reporting no difference in operative times [27, 28] and others reporting increased operative times for navigated TKA [17]; as a result, there is widespread perception that such technologies may lengthen operative time and are associated with a learning curve. Previous studies have defined learning curve as increased operative time immediately after introduction of the CAS followed by progressively decreasing operative times as surgeons acclimate to using the technology [27, 28]. Over the present study period, average operative time for the first cohort of cases using CAS increased non-significantly for two of three surgeons and decreased for the third. For the two surgeons with initial operative time increases, operative time decreased back toward baseline within the first 10 procedures, which aligns with learning curve trends reported in prior studies [23, 29]. Overall, operative times for navigated
Table 5 Combined nonnavigated TKA vs. initial CASassisted TKA and most recent CAS-assisted TKA

\begin{tabular}{lllll}
\hline $\begin{array}{l}\text { Control combined } \\
\text { cohort }\end{array}$ & $\begin{array}{l}\text { Initial navigated com- } \\
\text { bined cohort }\end{array}$ & $p$ value & $\begin{array}{l}\text { Most recent navigated } \\
\text { combined cohort }\end{array}$ & $p$ value \\
\hline $99.8 \pm 17.9 \mathrm{~min}$ & $104.1 \pm 10.4 \mathrm{~min}$ & 0.20 & $102.0 \pm 14.7 \mathrm{~min}$ & 0.55 \\
\hline
\end{tabular}


procedures decreased modestly over the course of the study, with average operative time actually falling below conventional baseline in some cases by the end of the study period.

Our results suggest that the learning curve associated with introduction of a novel imageless CAS may not be as steep as is commonly perceived, and that operative time increases during and after the acclimation period may, in practice, have minimal effect on practice economics or operative efficiency, as all differences were non-significant. Recent studies support these findings. Saiki et al. report that the learning curves between a different imageless CAS and an acceleration-based navigation system for TKA converged within five cases after introduction of these systems into surgical workflow, with ultimate average surgical times of $87.8 \mathrm{~min}$ and $87.4 \mathrm{~min}$, respectively [30]. Meyer et al. report that mean surgery duration for navigated TKA was 6 min longer than for conventional TKA [31]. These minimal increases may be enabled by standardization of navigation integration into surgical workflow, as well as ease-of-use characteristics inherent to a specific navigation system [31].

In addition, once the learning curve has been completed, the advantages posed by intraoperative navigation may decrease operative time as compared to conventional procedures. This is significant, because increased operative time has been cited as one of the main disadvantages associated with CAS [14]. These results are in line with a recent large database study by Sekimura et al., which reported initial increases in surgical time after introduction of TKA that declined over time based on aggregated data of TKA procedures utilizing a variety of CAS technologies [32]. However, to our knowledge this is the first study evaluating trends in learning curve and operative time for individual surgeons utilizing a single novel CAS, an approach previously used to assess the learning curve associated with robotic TKA technology [26].

The present study is not without limitations. Lengthening the study period may have allowed for further elucidation of trends in surgeon-specific learning curve; however, doing so would introduce cases conducted after the onset of the COVID-19 pandemic, for which operative time may be impacted by institutional protocols associated with pandemic control. In addition, patients were not randomized, but the sequential approach to recruitment enabled assessment of temporal trends in operative time. Finally, only three surgeons' experiences were captured as part of this study. Future studies should assess additional surgeon experiences to provide a more comprehensive understanding of the learning curve associated with navigated TKA.

In conclusion, the results of this study suggest that learning curve associated with a novel imageless CAS may be less steep than is commonly perceived, and any actual increases in operative time may be minimal when interpreted in a clinical context. This suggests that a novel imageless CAS may represent an easily integrated tool for improving alignment outcomes associated with TKA.

Funding No funding was received for this study.

\section{Declarations}

Conflict of interest RS has received consultancy fees from Intellijoint Surgical, Inc. and holds stock or stock options. SEM has received consultancy fees from Intellijoint Surgical, Inc. and holds stock or stock options. MM has received consultancy fees from Intellijoint Surgical, Inc.

Ethical approval IRB waiver for analysis of existing data.

Informed consent Not applicable.

\section{References}

1. Fingar KR, Stocks C, Weiss AJ et al (2006) Most frequent operating room procedures performed in U.S. hospitals, 2003-2012: statistical brief \#186. Healthcare cost and utilization project (HCUP) statistical briefs [Internet]. Agency for Healthcare Research and Quality (US), Rockville (MD)

2. Feng JE, Novikov D, Anoushiravani AA, Schwarzkopf R (2018) Total knee arthroplasty: improving outcomes with a multidisciplinary approach. J Multidiscip Healthc 11:63-73

3. Steinhaus ME, Christ AB, Cross MB (2017) Total knee arthroplasty for knee osteoarthritis: support for a foregone conclusion? HSS J 13(2):207-210

4. Kurtz S, Ong K, Lau E et al (2007) Projections of primary and revision hip and knee arthroplasty in the United States from 2005 to 2030. J Bone Jt Surg Am 89(4):780-785

5. Krackow KA, BayersThering M, Phillips MJ et al (1999) A new technique for determining proper mechanical axis alignment during total knee arthroplasty: progress toward computer-assisted TKA. Orthopedics 22(7):698-702

6. Antonios JK, Korber S, Sivasundaram L et al (2019) Trends in computer navigation and robotic assistance for total knee arthroplasty in the United States: an analysis of patient and hospital factors. Arthroplast Today 5(1):88-95

7. Ritter MA, Davis KE, Meding JB et al (2011) The effect of alignment and BMI on failure of total knee replacement. J Bone Jt Surg Am 93(17):1588-1596

8. Walker LC, Clement ND, Ghosh KM et al (2018) What is a balanced knee replacement? EFORT Open Rev 3:614-619

9. Czurda T, Fennema P, Baumgartner M et al (2010) The association between component malalignment and post-operative pain following navigation-assisted total knee arthroplasty: results of a cohort/nested case-control study. Knee Surg Sports Traumatol Arthrosc 18(7):863-869

10. Stucinskas J, Robertsson O, Sirka A, Lebedev A, Wingstrand H, Tarasevicius S (2015) Moderate varus/valgus malalignment after total knee arthroplasty has little effect on knee function or muscle strength. Acta Orthop 86(6):728-733

11. Werner FW, Ayers DC, Maletsky LP, Rullkoetter PJ (2005) The effect of valgus/varus malalignment on load distribution in total knee replacements. J Biomech 38(2):349-355 
12. Kim KT, Lee S, Ko DO, Seo BS, Jung WS, Chang BK (2014) Causes of failure after total knee arthroplasty in osteoarthritis patients 55 years of age or younger. Knee Surg Relat Res 26(1):13-19

13. Choong PF, Dowsey MM, Stoney JD (2009) Does accurate anatomical alignment result in better function and quality of life? Comparing conventional and computer-assisted total knee arthroplasty. J Arthroplasty 24(4):560-569

14. Jones CW, Jerabek SA (2018) Current role of computer navigation in total knee arthroplasty. J Arthroplasty 33(7):1989-1993

15. Foley KA, Muir JM (2019) Improving accuracy in total knee arthroplasty: a cadaveric comparison of a new surgical navigation tool, Intellijoint KNEE, with computed tomography imaging. Intellijoint Surgical Inc, Waterloo

16. Hsu RW, Hsu WH, Shen WJ, Hsu WB, Chang SH (2019) Comparison of computer-assisted navigation and conventional instrumentation for bilateral total knee arthroplasty: the outcomes at mid-term follow-up. Medicine (Baltimore) 98(47):e18083

17. Ajwani SH, Jones M, Jarratt JW et al (2012) Computer assisted versus conventional total knee replacement: a comparison of tourniquet time, blood loss and length of stay. Knee 19(5):606-610

18. Bae DK, Song SJ (2011) Computer assisted navigation in knee arthroplasty. Clin Orthop Surg 3(4):259-267

19. Bauwens $K$, Matthes $G$, Wich $M$ et al (2007) Navigated total knee replacement: a meta-analysis. J Bone Jt Surg 89(2):261-269

20. Shi J, Wei Y, Wang S et al (2013) Computer navigation and total knee arthroplasty. Orthopedics. https://doi.org/10.3928/0147744720131219-15

21. Cheng H, Clymer JW, Chen BP et al (2018) Prolonged operative duration is associated with complications: a systematic review and meta-analysis. J Surg Res 229:134-144

22. Hopper AN, Jamison MH, Lewis WG (2007) Learning curves in surgical practice. Postgrad Med J 83:777-779

23. Jenny J, Miehlke RK, Giurea A (2008) Learning curve in navigated total knee replacement. A multi-centre study comparing experienced and beginner centres. Knee 15(2):80-84
24. Ramsay C, Grant A, Wallace S et al (2000) Assessment of the learning curve in health technologies: a systematic review. Int $\mathbf{J}$ Tech Assess Health Care 16(04):1095-1108

25. Jenny J, Picard F (2017) Learning navigation-learning with navigation. A review. SICOT J 3:39

26. Sodhi N, Khlopas A, Piuzzi NS et al (2018) The learning curve associated with robotic total knee arthroplasty. J Knee Surg 31:17-21

27. Liodakis E, Antoniou J, Zukor DJ et al (2016) Navigated vs conventional total knee arthroplasty: is there a difference in the rate of respiratory complications and transfusions? J Arthroplasty 31(10):2273-2277

28. Gholson JJ, Duchman KR, Otero JE et al (2017) Computer navigated total knee arthroplasty: rates of adoption and early complications. J Arthroplasty 32(7):2113-2119

29. Smith BRK, Deakin AH, Baines J et al (2010) Computer navigated total knee arthroplasty: the learning curve. Comput Aided Surg 15:1-3

30. Saiki Y, Ojima T, Kabata T et al (2021) Accuracy of different navigation systems for femoral and tibial implantation in total knee arthroplasty: a randomised comparative study. Arch Orthop Trauma Surg 141:2267-2276

31. Meyer M, Renkawitz T, Völlner F et al (2021) Pros and cons of navigated versus conventional total knee arthroplasty-a retrospective analysis of over 2400 patients. Arch Orthop Trauma Surg 141:1983-1991

32. Sekimura TK, Upfill-Brown A, Hsiue PP et al (2021) Trends in operative time and short-term outcomes after conventional and navigated total knee arthroplasty. Arthoplasty Today 8:188-193

Publisher's Note Springer Nature remains neutral with regard to jurisdictional claims in published maps and institutional affiliations. 